\title{
GALAXY INTERACTIONS IN THE LOCAL VOLUME
}

\author{
I.D. KARACHENTSEV AND D.I. MAKAROV \\ Special Astrophysical Obs., Russian Academy of Sciences \\ N.Arkhyz, Stavropolsky kraj, KChR, 357147, Russia
}

\section{Introduction}

Imaging of a large number of faint galaxies with the Hubble Space Telescope reveals a significant excess of interacting and peculiar objects among them (Griffiths et al., 1994, Burkey et al., 1994). Being expected in general, this result needs, however, to be expressed quantitatively. To estimate evolutionary changing of the relative number of interacting galaxies, $\delta_{\text {int }}(\mathrm{z})$, from their redshift $z$, one must determine reliably the local value, $\delta_{\text {int }}(0)$. But due to a subjective manner of definition of interacting galaxy system, the published values of $\delta_{\text {int }}(0)$ differ from one author to another several times. The most appropriate sample to estimate $\delta_{\text {int }}(0)$ would be quite a representative sample of very nearby galaxies, which is restricted by their distance or radial velocity. Below we consider data on such a fair sample, named the Local Volume $(=\mathrm{LV})$, which is the most complete one at the present moment.

\section{Basic properties of the Local Volume sample}

Kraan-Korteweg \& Tammann (1979) compiled a catalog of nearby galaxies having radial velocities corrected for the solar motion within $500 \mathrm{~km} / \mathrm{s}$. After avoiding of probable Virgo cluster members, their sample consisted of 179 galaxies. Later the list of LV galaxies was updated essentially in three different ways: a) accidental revealing of new dwarf galaxies with radial velocities below $500 \mathrm{~km} / \mathrm{s}$ during optical and HI surveys of the known catalogs; b) HI observations of new galaxies which have been found in a strong extinction zone along the Milky Way; c) special searches for and subsequent HI surveys of dwarf galaxies in the vicinity of nearby groups.

As a result, at the present moment the population of the LV increased up to 277 galaxies, including 255 galaxies with $V_{0}<500 \mathrm{~km} / \mathrm{s}$, and 22 

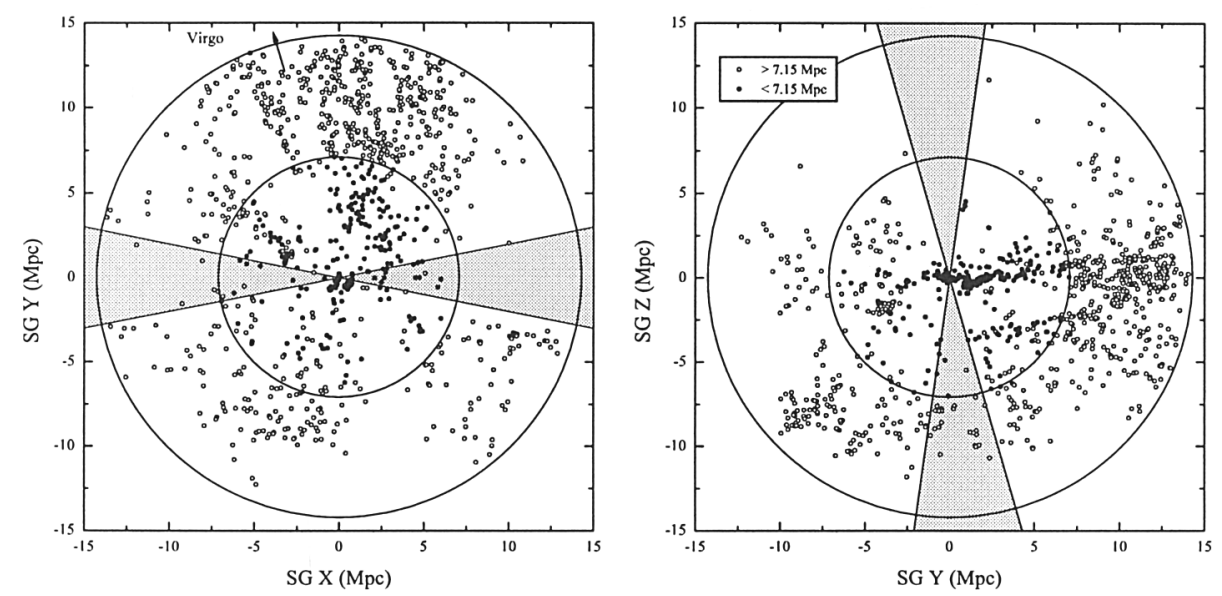

Figure 1. Distribution of nearby galaxies in the Supergalactic Cartesian coordinates. Filled circles are galaxies with a distance $D<7.15 \mathrm{Mpc}$, open circles are more distant ones with $V_{0}<1000 \mathrm{~km} / \mathrm{s}$. The shaded cone indicates an absorption zone. The arrow shows a direction toward the Virgo cluster.

spheroidal dwarf systems, which have no radial velocities but are probable companions of the known nearby galaxies.

We should emphasize that besides its numerical growth the LV sample is enriched strikingly by data on galaxy distances. As it is known, due to coherent and random non-Hubble motions the radial velocity of a nearby galaxy is rather unreliable indicator of its distance. During the 1990s in the Special Astrophysical Observatory there was undertaken a project of CCD imaging of more then 100 nearby galaxies with the goal of measuring their distances via the brightest stars. Together with data obtained at other observatories the number of the LV galaxies having direct (photometric) distance estimates has reached 158. Among the rest of the LV galaxies 35 are with distances adopted according to their membership in the known groups, and only 84 have kinematic distances, $D=V_{0} / H$, derived under the local Hubble parameter $H=70 \mathrm{~km} / \mathrm{s} / \mathrm{Mpc}$ (Karachentsev \& Makarov, 1996). Note that in the initial sample of Kraan-Korteweg \& Tammann (1979) the photometric distance estimates were known for 15 galaxies only. Therefore, the present 3-D structure of the LV looks much more detailed than decade ago.

Fig. 1 shows the LV galaxy distribution (filled circles) in projection onto the Local Supercluster plane and also edge-on. More distant galaxies with radial velocities $V_{0}<1000 \mathrm{~km} / \mathrm{s}$ are indicated with open circles. The region of strong extinction in the Milky Way is shaded.

Distribution of the LV galaxies by their angular diameters and distances 


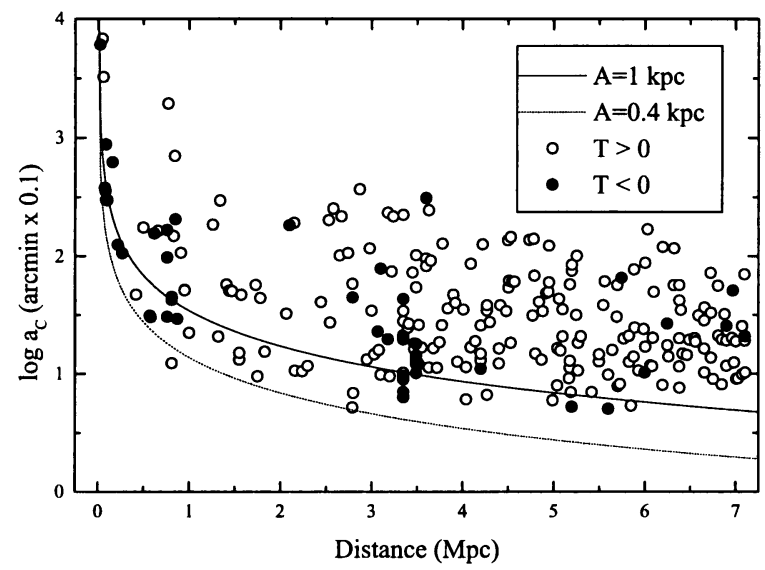

Figure 2. Distribution of the LV galaxies vs. their distance and angular diameter, corrected for inclination and absorption. The lines correspond to the linear diameter of $1 \mathrm{Kpc}$ and $0.4 \mathrm{Kpc}$. The early type galaxies are indicated by filled circles.

is shown in Fig. 2. Two lines in it correspond to the values of linear diameter: $1.0 \mathrm{Kpc}$ and $0.4 \mathrm{Kpc}$. As one can see from the diagram, at distances $D>4$ $\mathrm{Mpc}$ there is a deficit of galaxies with small linear diameters. The data of Fig. 2 allow us to judge the completeness of our sample. A simple analysis yields the completeness level to be about $70 \%$. Taking into account this correction, the mean number density of the Local Volume is $0.25 \mathrm{gal} / \mathrm{Mpc}^{3}$.

\section{The nearby interacting galaxies}

The most systematic searches for interacting galaxies were undertaken by Vorontsov-Velyaminov $(1959,1977)$, who published the Atlas of 850 interacting galaxies, and also by Arp (1966), whose Atlas contains images of 338 peculiar and interacting objects. Among 603 isolated pairs of galaxies in the northern sky 314 interacting system were noted in his Catalogue by Karachentsev (1987). Vorontsov-Velyaminov's survey was based on an inspection of the Palomar Observatory Sky Survey prints, whereas Arp used high resolution photographs obtained on the $200^{\prime \prime}$ telescope.

In Table 1 we collected all cases when a galaxy from the Local Volume is present in the Atlases of Vorontsov-Velyaminov $(=\mathrm{VV})$ or Arp. First three columns contain the galaxy common name and its number in both Atlases, the forth one indicates the value of "Tidal Index" described below, and the last column gives brief remarks concerning interaction features. Basing on these data and taking into account the incompleteness of both the surveys in the southern sky, we conclude that the local value of the relative abundance of interacting galaxies is $\delta_{\text {int }}(0)=(5-6) \%$. However, we 
should note that the cases when a galaxy is classified as interacting by both the authors seen to be too infrequent. This may be caused by prevalence of a subjective factor when classifying signs of interaction even for the nearest galaxies. In many cases the irregular patchy shape of dwarf galaxies gives an erroneous impression of an interacting system. In fact, judging by remarks in Table 1, only two galaxies, M 101 and its companion NGC 5474, may be considered as actually interacting system with tidal distortions of their spiral structures. Therefore, when the single objects of irregular shape are excluded, the estimate of $\delta_{\text {int }}(0)$ drops to $\sim 1 \%$.

TABLE 1. Nearby interacting and peculiar galaxies.

\begin{tabular}{|c|c|c|c|c|}
\hline Name & VV & Arp & $\Theta$ & Remarks \\
\hline M 32 & - & 168 & 5.97 & Compact \\
\hline N 1313 & 436 & - & -1.58 & Irregular \\
\hline N 1569 & - & 210 & 0.36 & MERGER? double nuclei \\
\hline N 2537 & 138 & 6 & -1.58 & Irregular,HSB \\
\hline Holmb.II & - & 268 & 0.61 & Irregular \\
\hline DDO 53 & 499 & - & 0.56 & Irregular \\
\hline M 81 & - & - & 1.01 & MERGER, Arp's loop \\
\hline M 82 & - & 337 & 2.30 & MERGER? \\
\hline N 3077 & - & 一 & 2.27 & MERGER, "Garland" \\
\hline VII Zw403 & 574 & - & -0.08 & Irregular \\
\hline N 3738 & - & 234 & -0.40 & Irregular \\
\hline N 4190 & 104 & - & 0.19 & Irregular \\
\hline P 42134 & 42 & 211 & -1.04 & Irregular \\
\hline GR 8 & 558 & - & -1.12 & Irregular \\
\hline N 5128 & - & 153 & 0.38 & MERGER! \\
\hline N 5238 & 828 & 一 & -0.58 & Irregular \\
\hline $\mathrm{U} 8638$ & 133 & - & -1.15 & Irregular \\
\hline M 101 & 344 & 26 & 0.70 & Mutually interacting \\
\hline N 5474 & 344 & 26 & 1.91 & Mutually interacting \\
\hline N 5477 & 561 & - & 1.48 & Irregular \\
\hline Milky W. & - & - & 2.71 & MERGER,polar Magell. ring \\
\hline N 6946 & - & 29 & -1.35 & Irregular arm \\
\hline
\end{tabular}

Beside $\delta_{\text {int }}(0)$, the local relative number of merging galaxies, $\delta_{\text {merg }}(0)$, is of evolutionary interest too. We did not find any estimate of this quantity in literature. Based on large scale photographs and CCD frames available almost for all the LV galaxies, we may rank among mergers the six nearby galaxies indicated in Table 1. Among them there is a classic merger example, 
NGC $5128=$ Centaurus A, and also our Galaxy with its polar Magellanic ring. Therefore, in a first approximation one may adopt $\delta_{\text {merg }}(0)=(2-3) \%$ as the local abundance of mergers. Note, that signs of merging, as well as interaction, are seen more frequently among the most luminous galaxies. For double galaxies this effect was described by Karachentsev (1987).

\section{Tidal index as a measure of interaction}

For objective description of interaction we use a quantitative approach, where each LV galaxy $i$ has a tidal index

$$
\Theta_{i}=\max \left\{\log \left(M_{k} / D_{i k}^{3}\right)\right\}+C, \quad k=1,2 \ldots N .
$$

Here $M_{k}$ and $D_{i k}$, are mass and 3D separation of a neighboring galaxy. In such a way we find for each galaxy its "Main Disturber" (=MD), which produces the highest tidal action on the galaxy. Calculating the $\Theta$ index we also took into account more distant galaxies with radial velocities up to $1000 \mathrm{~km} / \mathrm{s}$ (see Fig. 1). The value of the arbitrary constant $C$ was taken from the following condition. A galaxy, which is interacting with its MD, may be described with a cyclic Keplerian period

$$
t_{i k}=D_{i k}^{2 / 3} \cdot G^{-1 / 2} \cdot\left(M_{i}+M_{k}\right)^{-1 / 2},
$$

where $G$ is the gravity constant. We determine the value of $C$ from the requirement that $\Theta$ is zero when the Keplerian period equals cosmological time, $1 / H$. In the units of $\left\{M_{\odot}, \mathrm{Mpc}\right\}$ this gives $C=-11.75$. Using the idea of critical density, $\rho_{\mathrm{c}}=3 H^{2} / 8 \pi G$, one can express the condition $\Theta$ $=0$ in another way: $\rho_{k}(\Theta=0)=\rho_{\mathrm{c}}$, where $\rho_{k}$ means a density excess, caused by the MD with a mass $M_{k}$ at a distance $D_{i k}$. Therefore, we have a reason to consider a galaxy with tidal index $\Theta<0$ a rather isolated one, which is causatively unrelated to its neighbors. As is seen from Table 1, the majority of irregular galaxies which have been classified as interacting by Vorontsov-Velyaminov and Arp are actually isolated objects.

The total mass of a galaxy was determined as $M=\kappa V_{\mathrm{m}}^{2} \cdot A_{25} / 2 G$, where $V_{\mathrm{m}}$ is the galaxy rotation curve amplitude derived from its $\mathrm{HI}$ line width after correction for inclination and turbulent velocity in the manner described in PGC-ROM (Paturel et al., 1996). The dimensionless constant $\kappa$ is adopted to be 2.5 in order to take into consideration that the typical rotation curve of a galaxy extends 2.5 times further than its standard optical radius (Broeils, 1992). In the case of a galaxy with the unknown $\mathrm{HI}$ linewidth or with the inclination angle $i<40^{\circ}$ its mass estimate was made via luminosity $L$ taking into account its morphological type: $\left(M / M_{\odot}\right)=\kappa \cdot(8-0.4 \cdot T) \cdot\left(L / L_{\odot}\right)$. This kind of mass estimate was applied 
to $46 \%$ of the LV sample. The general list of the galaxies in the Local Volume with indication of their distances, masses, tidal indices, and the Main Disturber names was published in Karachentsev \& Makarov (1997). The updated version of the list is accessible via e-mail from dim@sao.ru.

\section{The tidal index and other properties of the LV sample}

Fig. 3 reproduces a distribution of the number of the LV galaxies vs. their tidal index. The histogram of $N(\Theta)$ has an asymmetric shape with a maximum at $\Theta \simeq-1$. More than half of the galaxies (55\%) are situated in the region of $\Theta<0$, i.e. they may be considered as rather isolated objects. For them the $\Theta$-parameter has rather a sense of isolation index than tidal. The galaxies of early types, $T<0$, are indicated on the histogram by a gray area. On the average they have higher values of the $\Theta$-index, which demonstrates the existence in the LV of the known effect of segregation of elliptical and spiral galaxies depending on the density of their environment.

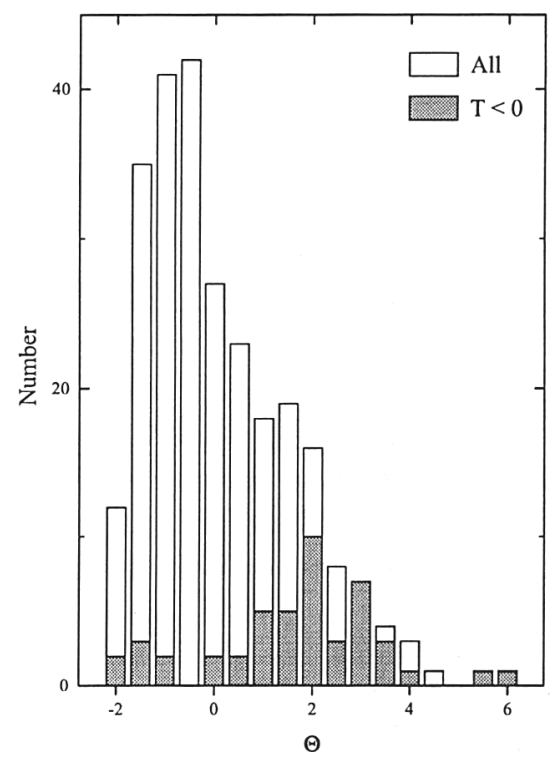

Figure 3. Distribution of the LV galaxies on their Tidal Index, $\Theta$. The early type objects are shaded.

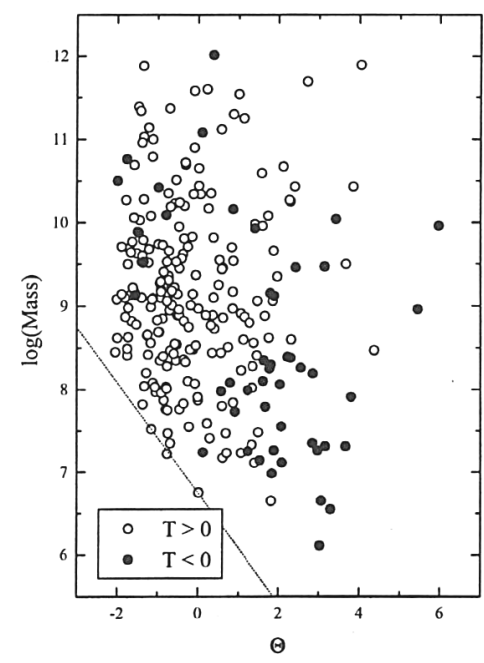

Figure 4. Masses of the LV galaxies versus their Tidal Indices. Early and late galaxy types are indicated by filled and open circles. An empty lower left corner is caused by the Milky Way "shadow".

The distribution of the LV galaxiez vs. their masses and tidal indexes (Fig. 4) shows that these values are practically uncorrelated. The dotted line in the lower left corner indicates a zone of observational selection, where isolated objects of low mass are absent due to the Milky Way influence. With correction of this feature the distribution of $\{M, \Theta\}$ does not find 
any noticeable segregation of the galaxies by their masses depending on the number density of neighbors. However, it attracts attention that on the diagram the galaxies of early and late types (filled and open circles) have essentially different distribution. All dwarf elliptical and spheroidal galaxies with $\log M<9$ have $\Theta>0$, i.e. they occur only in dense regions around massive MDs. The observed absence of isolated objects among spheroidal dwarfs may be considered as an argument in favor of their origin from HI rich irregular dwarf systems with tidal evolution effects.

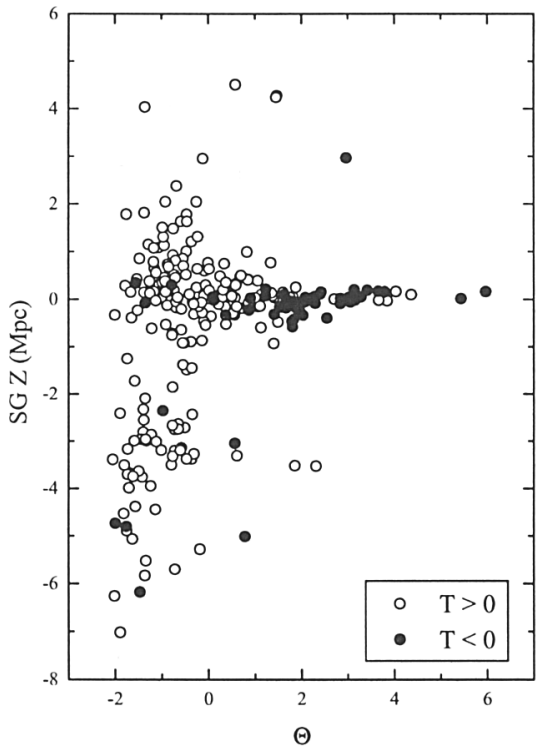

Figure 5. Map of Tidal Index of the LV galaxies versus their distance from the $\mathrm{Su}$ pergalactic plane. E+Sph and S+Irr types are shown with different symbols.

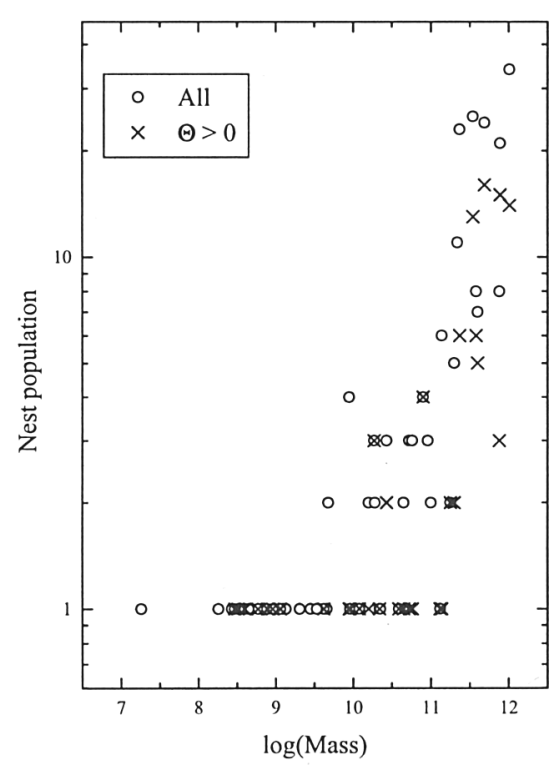

Figure 6. Number of galaxies in a nest around Main Disturber versus its mass. Circles are the total nest population, crosses are the same with $\Theta>0$.

Fig. 5 presents the distribution of the LV galaxies vs. their tidal indices and distances from the Local Supercluster plane. As it is seen from the data, the early type galaxies (filled circles) are concentrated toward the LS plane stronger than spiral and irregular ones, which confirms again the existence of the effect of morphological segregation within the Local Volume. One can also note another feature. Among the galaxies having a high (positive) tidal index more than $90 \%$ of them are situated in a narrow layer, $|\mathrm{SGZ}|<0.5$ $\mathrm{Mpc}$, while isolated objects spread throughout the Local Volume. Such a feature may indicate the presence of two subsystems in the LV: "disc" and "bulge", where conditions for galaxy interaction are very different.

A relative number of galaxies, which are the Main Disturbers for one or several neighbors, make up $24 \%$. This value is defined by the properties 
of the 3D structure of the LV, as well by the galaxy mass function. Being ordered according to their MDs, the LV galaxies form "nests" with a population $k$ from 1 to 34 members. Obviously, the nest population must be higher the more massive its MD is. Such a relation is actually seen in Fig. 6, where the nests (open circles) have a tendency to follow the line $k \sim M^{1 / 2}$ . In particular, the seven most massive galaxies control $51 \%$ of the total LV population within their zones of gravitational influence.

However, we should remark that the majority of nest members around MDs have tidal indices $\Theta<0$, i.e. their crossing time with respect to the MD exceeds the cosmological time, $1 / H$. If one takes into consideration as real companions of MDs only the galaxies with $\Theta>0$ (crosses in Fig. 6), the typical nest population drops about 2 times.

In conclusion we note that the Local Volume contains some objects, not distinguished by signs of peculiarity or interaction, which nevertheless need special explanations. As an example we mention the case of NGC 404. This compact, bright lenticular galaxy has the corrected radial velocity $V_{0}$ $=+195 \mathrm{~km} / \mathrm{s}$ or kinematic distance of $2.8 \mathrm{Mpc}$. Within $\sim 2 \mathrm{Mpc}$ around it there are no other galaxies. NGC 404 has a rather low tidal index, $\Theta=$ -0.78 , and its MD is M 31. Judging by its HI flux, NGC 404 is moderately rich in neutral gas, and also several dust clouds are seen near its nucleus. The origin of such a very isolated compact galaxy seems a puzzle. Probably, the galaxy is the final stage of consecutive merging of members of the former group. Such kind of nearby unusual objects deserve the closer attention of observers as well as theorists.

\section{Acknowledgements}

This work is supported by INTAS-RFBR grant No 95-IN-RU-1390, and RFBR grant No 97-02-27101.

\section{References}

Arp H. (1966) Atlas of Peculiar Galaxies, Asroph. J. Suppl., 14, p.1

Broeils A. (1992) Dark and Visible Matter in Spiral Galaxies, Dissertation, Groningen Burkey J.M., Keel W.C., Windhorst R.A., Franklin B.E. (1994) Asrophys.J., 429, p.413 Griffiths R.E., Casertano S., Ratnatunga K.U. et al. (1994) Astrophys.J., 435, L19

Karachentsev I.D. (1987) Binary galaxies, Moscow, Nauka

Karachentsev I., Makarov D. (1996), Astron.J., 111, p.794

Karachentsev I., Makarov D. (1997), Astrophysical Letters \& Communications, accepted Kraan-Korteweg R.C., Tammann G.A. (1979), Astron.Nachr, 300, p.181

Paturel G., Bottinelli L., Di Nella H. et al. (1996) Principal Galaxy Catalogue, PGCROM, Observatoire de Lyon

Vorontsov-Velyaminov B. (1959) Atlas and Catalogue of Interacting Galaxies, Part I, Moscow Univ.

Vorontsov-Velyaminov B. (1977), Astron. Asrtophys. Suppl., 28, p.1 